\title{
Examination of heavy-ion collisions using EPOS model in the frame of BES program
}

\author{
Maria Stefaniak
}

\begin{abstract}
EPOS is a generator which allows one to simulate various types of collisions of divers systems with different initial parameters. It considers the parton-based Gribov-Regge theory. So far the EPOS model has been used to describe higher collision energies obtained with RHIC ${ }^{1}$ or LHC $^{2}$ data. On the other hand, there is another program under investigation: Beam Energy Scan conducted at Brookhaven National Laboratory. The beams of gold ions are collided at energies such as $\sqrt{s_{N N}}-7.7,11.5,19.6,27,39$, and $62.4 \mathbf{~ G e V}$ in order to collect and analyze data needed in examination of $\mathbf{Q G P}^{3}$ Phase Diagram. The EPOS model could become a useful tool in such studies. Simulated with EPOS data will be verified using elliptic flow analyses and two-particles femtoscopic correlations, which allow one to measure the size of sources determined by newly created particle.
\end{abstract}

\section{INTRODUCTION}

Studying the properties of the nuclear matter is an extremely difficult and complex challenge. In order to achieve conditions needed to create systems containing particles we are looking for the heavy-ions are collided in major experiments. The information about newly-created matted are collected in detectors and analyzed by experimental physicists. Scientists explain occurring processes in detailed theories. One of such descriptions is included in the EPOS model. It considers the pardon-based Gribov-Regge theory [1]. EPOS gives possibility to study different observables what helps to understand even complex reactions with heavy-ions. So far the EPOS model has been used to describe higher collision energies obtained with RHIC or LHC. On the other hand, there is another interesting program currently under investigation at RHIC: Beam Energy Scan (BES), conducted at Brookhaven National Laboratory. Main goals of this project are to examine the Phase Diagram, to study the characteristics of the first-order phase transition between Hadron Gas and Quark-Gluon Plasma phases of nuclear matter, to search signatures of turn-off the QGP. The search of Critical Point between first-order phase transition and transition of "cross-over" is also significant topic. The variety of initial conditions allows to cover as widest part of Phase Diagram of nuclear matter as possible.

\section{BEAM ENERGY SCAN}

Beam Energy Scan is a scientific project run on the RHIC complex. The main goal, besides investigation of properties of QGP, is understanding the QCD phase diagram structure (Fig.

M. Stefaniak is with Warsaw University of Technology, Warsaw, Poland, (e-mail: maria.stefaniak@fizyka.pw.edu.pl).

${ }^{1}$ Relativistic Heavy-Ion Collider

${ }^{2}$ Large Hadron Collide

${ }^{3}$ Quark Gluon Plasma
[1]). Only a few pieces of information have been discovered yet. The QCD phase diagram is being mapped out in order to look for the evidences of:

- critical point fluctuations

- turn-off the signatures of QGP

- signals of $1^{\text {st }}$ order phase transition [2]

The gold ions are collided with such energies as: $\sqrt{s_{N N}}=$ 7.7, 11.5, 19.6, 27, 39, $62.4 \mathrm{GeV}$. Femtoscopic correlations or analysis of elliptic flow are two of ways of studying transitions of matter.

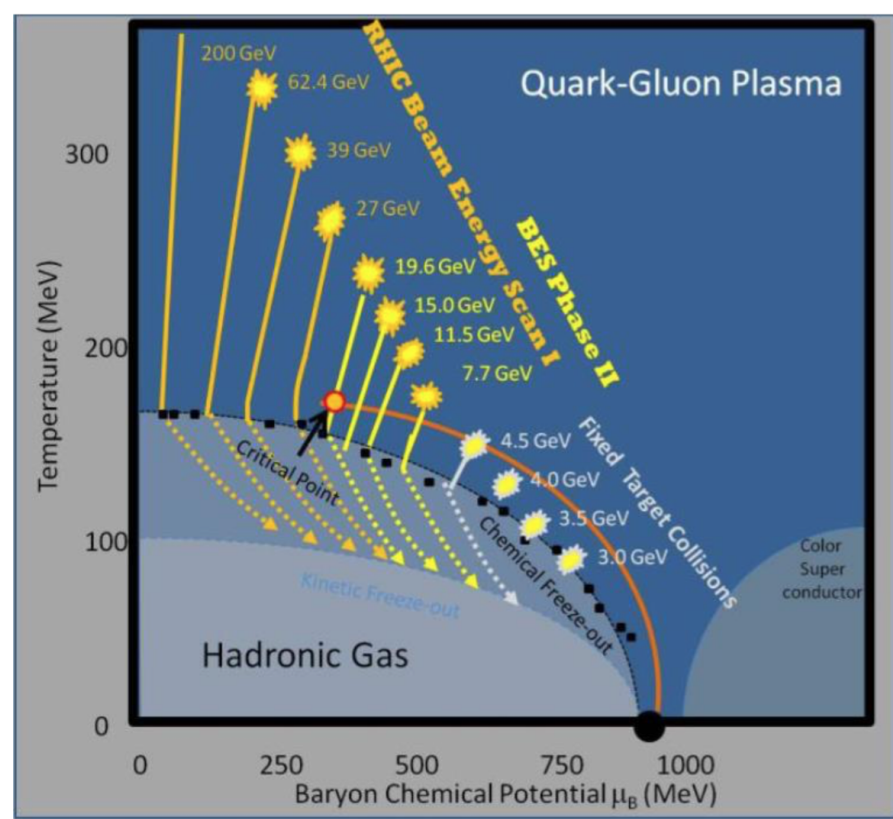

Fig. 1. Studies of phase diagram peformed in a frame of BES program [3]

\section{FEMTOSCOPY CORRELATIONS}

\section{A. Two-particle correlations}

The method inspired by HBT ${ }^{4}$ interferometry [4] considers the smallest sizes in nature, about $10^{-15} \mathrm{~m}$ (femtometre), the sizes of nuclei. Studies of two-particle correlations allows one to investigate space-time extension of the emitting source created during the collisions of nuclei. The size of area where the given kind of particles are produced is called homogeneity length [5].

${ }^{4}$ R. Hanbury-Brown and R. Q. Twiss 
1) Correlation function: A two-particle correlation function defines ratio between the probability of detecting two particles with given momenta and position simultaneously to the product of detecting them separately [5].

$$
C\left(p_{1}, p 2\right)=\frac{P_{2}\left(p_{1}, p_{2}\right)}{P_{1}\left(p_{1}\right) P_{1}\left(p_{2}\right)}
$$

The source is often treated as a three-dimensional sphere and described with parametrization proposed by G. Bertsch and S. Pratt [6] in Longitudinally Co-Moving System where: $p_{1, L}+p_{2, L}=0$. The correlation function is represented in the -out -side -long (o-s-1) coordinate system [7]. Kopylov and Podgoretsky proposed the three-vector $\vec{q}=\overrightarrow{p_{1}}-\overrightarrow{p_{2}}$ so that the correlation function is represented with the equation (assuming Gaussian source parametrization):

$$
\begin{gathered}
C\left(q_{\text {out }}, q_{\text {side }}, q_{\text {long }}, \lambda\right)= \\
1+\lambda \exp \left(-q_{\text {out }}^{2} r_{\text {out }}^{2}-q_{\text {side }}^{2} r_{\text {side }}^{2}-q_{\text {long }}^{2} r_{\text {long }}^{2}\right)
\end{gathered}
$$

where $r_{\text {out,side,long }}$ are lengths of the source in out, side and long directions (three-dimensional femtoscopy radii) and $\lambda$ is a chaosity parameter [Fig. 2].

In the three-dimensional analysis the values of the $R_{\text {out }}, R_{\text {side }}$ and $R_{\text {long }}$ are considered to be optimal in studies of the timespace characteristic of the particle-emitting source [8] [5].

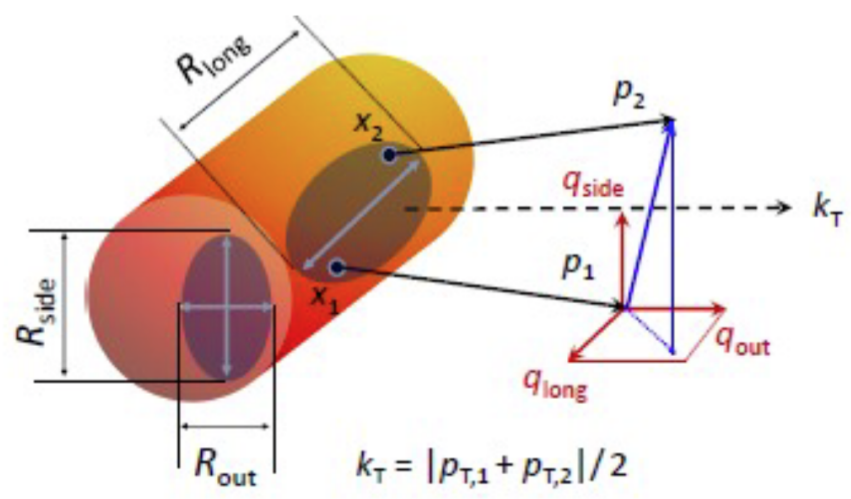

Fig. 2. Definition of the three-dimensional femtoscopy radii.

In analysis of the experimental data the function is parametrize with the following form:

$$
C_{2}(\vec{q})=\frac{A(\vec{q})}{B(\vec{q})}
$$

where A (signal) is a momentum distribution of particles built in the same event, while B (background) is a reference momentum distribution obtained from different events.

\section{AZIMUTHAL ANISOTROPY}

The azimuthal anisotropy characterizes expansion of highlycompressed matter created in heavy-ion collisions [Fig. 3]. It is one of the most significant tool used in studying hot and dense matter, described more detailed in Ref. 9.

The shape of the expansion could be expressed with the

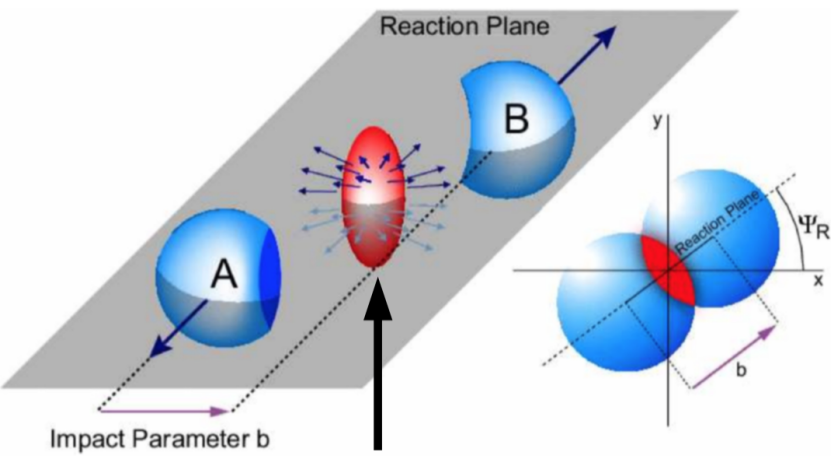

Fig. 3. Nucleus after collision. [10]

Fourier decomposition. The second order contribution responds to elliptic flow. One of the standard methods used in order to calculate the flow is event plane method. The crucial part is to estimate the reaction plane from the measured particles. It is defined as an event plane in range $\Psi_{n}\left(-\pi / n \geq \Psi_{n}<\pi / n\right)$. In order to calculate the event plane for elliptic flow one can use equation:

$$
\Phi_{2}=\tan ^{-1}\left(\frac{\sum_{i} w_{i} \sin \left(2 \phi_{i}\right)}{\sum_{i} w_{i} \cos \left(2 \phi_{i}\right)}\right) / 2
$$

where: $i$ - selected particle, $\phi_{i}$ - azimuthal angle of particles, $w_{i}$-weight of $i$ particle [11]. In proposed $\eta-s u b$ method, from all measured particles there are selected two groups with "forward" and "backward" pseudorapidity with a gap between them. This method is introduced with a view to reducing the effect of "non flow" (e.g. decay of resonances to several charged daughter particles, jets or HBT correlations). [12] To express the observed $v_{2}$ of particles with respect to already investigated event plane one uses:

$$
v_{2}^{o b s}\left(p_{T}, y\right)=\left\langle\cos \left[2\left(\phi_{i}-\Phi_{2}\right)\right]\right\rangle
$$

This is average over all particles in all events. As a consequence of final multiplicity limitation in the investigation of the angle of the reaction plane, the correction of $v_{2}$ with event plane resolution have to be done.

$$
R_{2}=\sqrt{\left\langle\cos \left[2\left(\Phi_{2}^{A}-\Phi_{2}^{B}\right)\right]\right\rangle}
$$

where: $\Phi_{n}^{A}$ - event plane calculated only using "forwardpseudorapidity" particles while $\Phi_{n}^{B}$ - with "backwardpseudorapidity" ones. The final result of $v_{2}$ calculation is given by:

$$
v_{2}=\frac{v_{2}^{o b s}}{R_{2}}
$$

\section{EPOS}

EPOS is a model which consists:

Energy conserving quantum mechanical multiple scattering approach, based on Partons (parton ladders), Off-shell remnants, and Splitting of parton ladders [1].

EPOS is a generator based on Parton-Based Gribov-Regge theory which is the mixture of two diverse theories. First 
one, Gribov-Regge uses effective field principies and it focuses on the soft aspects of particle collision. The interactions are described with theoretical objects called Pomerons, which are crucial in calculation the cross-section in multiple scattering (Fig. 4). The eikonalized parton model introduces quarks and gluons, what results with a more precise description of the interactions such as a gluon exchange. The $\mathrm{QCD}^{5}$ and $\mathrm{QED}^{6}$ are included in the description as well. [1] [13]

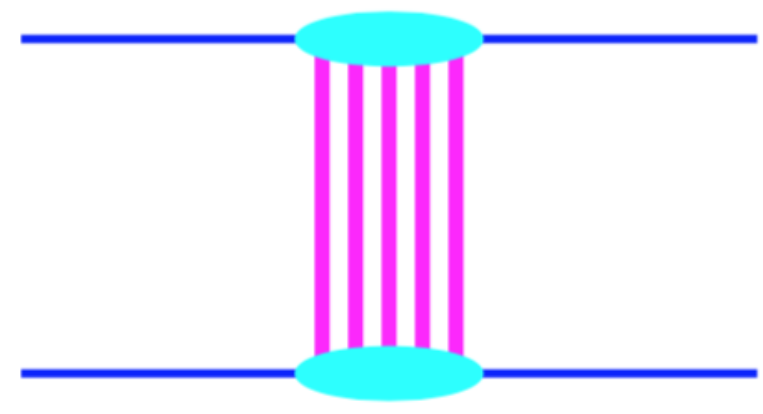

Fig. 4. Hadron - hadron multiple scattering. Navy blue lines are hadrons and each of pink line illustrate Pomeron. [1]

The combination of Gribov - Regge theory and parton model ensures that the energy is shared properly between both the particle production and the cross section. With the parton model there are hard processes added as well. The production of partons is represented by Lund model strings [14]. There are introduced objects called parton ladders. The open ones contribute to inelastic scattering which supports particles production. Meanwhile the closed ladders illustrates elastic scattering (Fig. 5). In third version of EPOS there is applied the procedure consists hydrodynamic effects described in Ref. 15.

\section{RESUlts}

Three different kinds of analysis were performed: oneparticle analyze $-p_{T}$ spectra, azimuthal anisotropy $-v_{2}$, two-particles analysis - femtoscopy correlations, in order to compare simulated and experimental data.

\section{A. Transverse momentum spectra}

Studies of the transverse momentum are the most elementary in High-Energy analysis. The obtained distributions are compared with the experimental data published in Ref. 16. Such measurements were done for $\mathrm{Au}+\mathrm{Au}$ collisions at two centrality ranges: $0-5 \%$ (central) and $60 \%-80 \%$ (peripheral events). Particles with rapidity $|y|<0.5$ were taken into consideration. Below there are placed the obtained $p_{T}$ distributions for $\frac{h_{+}+h_{-}}{2}$, the sum of positive-charged hadrons $\left(p, \pi^{+}, K^{+}\right)$and negative once $\left(\bar{p}, \pi^{-}, K^{-}\right)$.

There is high agreement between experimental and theoretical data registered for the most central collisions. However, for lower energies for higher values of $p_{T}$ the slopes of plotted

${ }^{5}$ Quantum Chromodynamics

${ }^{6}$ Quantum Electrodynamic a)

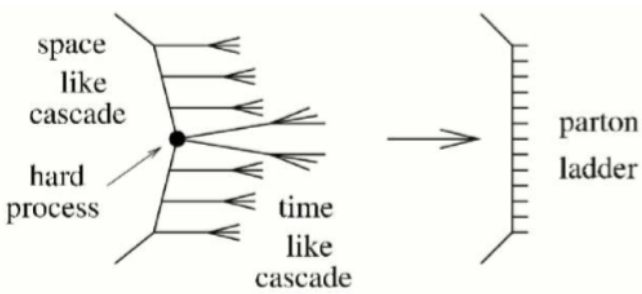

b)
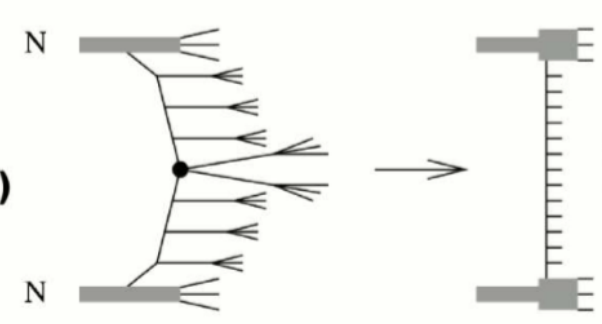

projectile remnant excitation

arton ladder

target

remnant excitation

c)

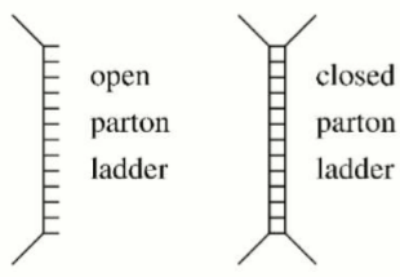

Fig. 5. a) Elementary parton - parton scattering and representation as parton ladder. b) Full presentation of scattering, completed with remnants. c) Open and closed parton ladder [13]

data are slightly different. The strong expansion of the matter in the transverse direction is too low in comparison with the experimental results. In case of peripheral collisions the difference is more visible.

\section{B. Correlation function}

There was performed positively charged pions femtoscopic analysis in $\mathrm{Au}+\mathrm{Au}$ collisions. In order to study beam energy in femtoscopy the were calculated and parametrized threedimensional correlation functions. The $\pi^{+}$pairs were taken into consideration at $\sqrt{s_{N N}}=11.5 \mathrm{GeV} 19.6$ and $39 \mathrm{GeV}$ at different centralities or $k_{T}$ ranges. With a view to inferring size of emitting source the fitting procedure was used (equation 2). Obtained radii and $\lambda$ parameter from calculation of simulated data were compared with published experimental results and presented in different combinations.

With the decrease of energy of collision $\sqrt{s_{N N}}$ the differences becomes more relevant. Calculated values of $R_{\text {out }}$ are in the range of expectations. The duration of particle emission is similar for compared systems. In case of measurements of $R_{\text {side }}$ the experimental results are higher then the simulated once, especially for lower $k_{T}$ bins. The sizes of sources created in STAR are bigger then simulated in EPOS. However, the most visible discrepancy is in studies of $R_{\text {long }}$. In order to explain these differences the elliptic flow analyze was performed.

It is possible in EPOS generator to turn off some physics 

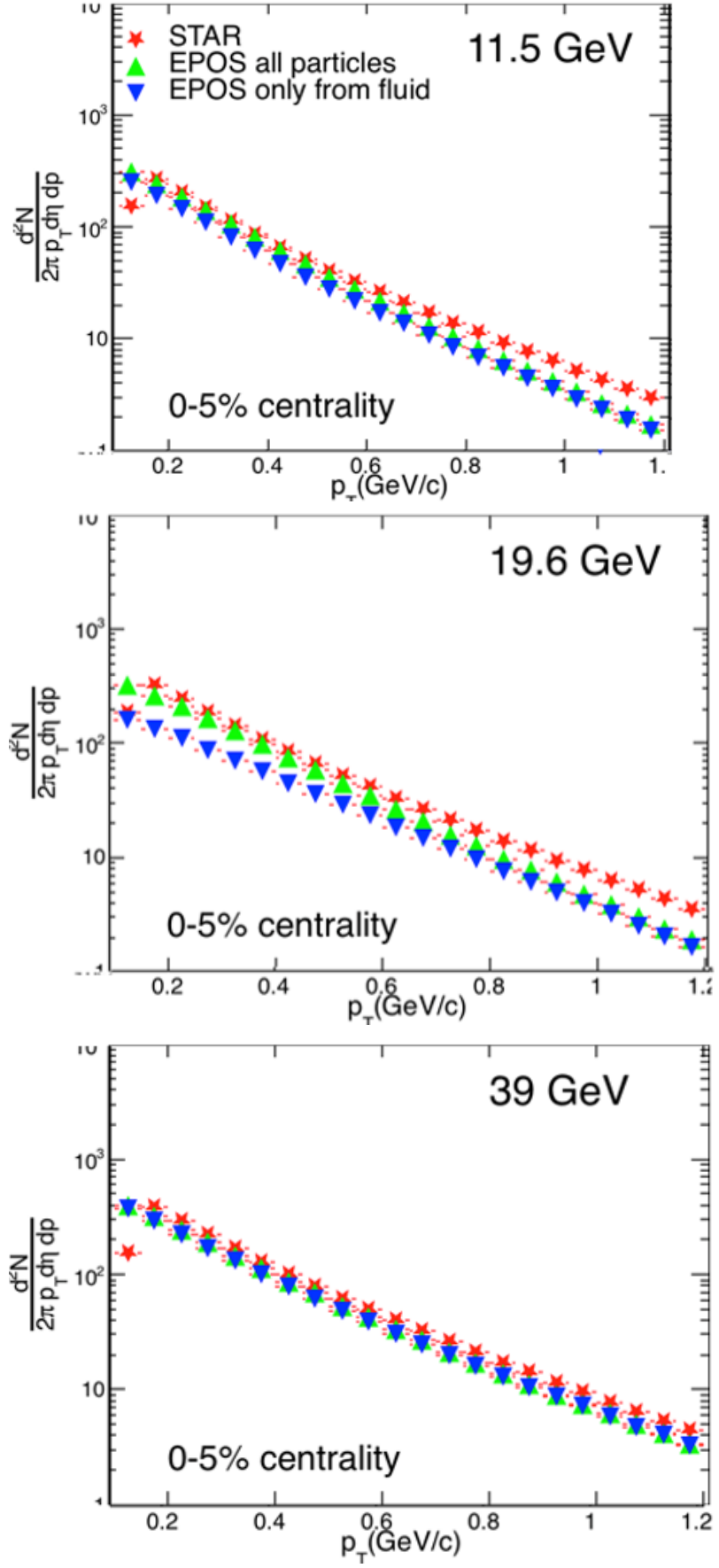

Fig. 6. $p_{T}$ spectra for energies $\sqrt{s_{N N}}=11.5,19.6,39 \mathrm{GeV}$ at $0-5 \%$ centrality range.

processes occur in heavy-ion collisions. In order to study the impact of hadron cascades on size of emitting source, they were turn off and turn on in simulation. The results are presented in Figure 8. Hadron cascades have relevant influence on values of calculated radii and $\lambda$ parameter. When they are present the size of the source is bigger and more similar to experimental studies. The $\lambda$ parameter and $R_{\text {out }}$ are in the range of expectations, while rest of parameters are considerably lower then in experimental results. Too high value of ratio $R_{\text {out }} / R_{\text {side }}$ means that the lifetime of the source is shorter for simulated collisions than those obtained in experiments.
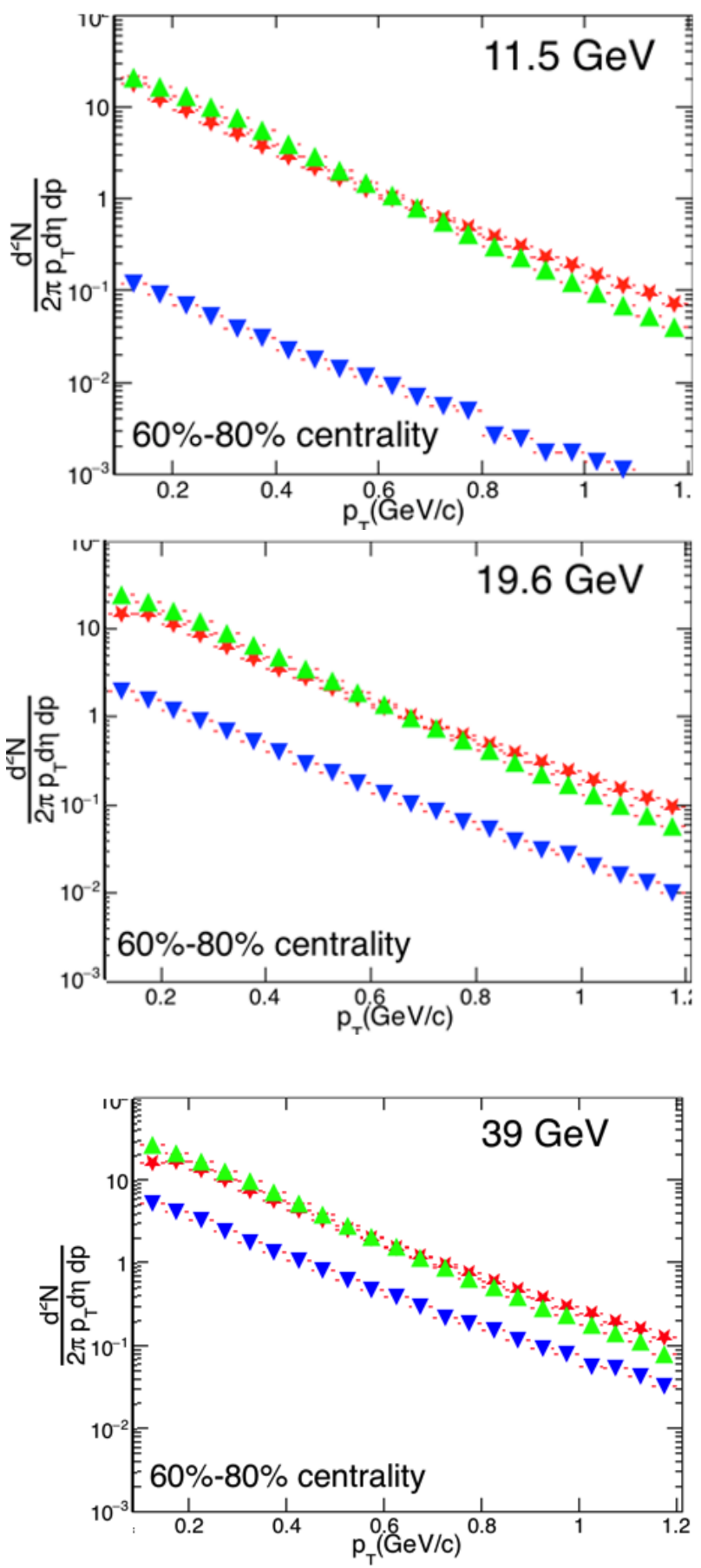

Fig. 7. $p_{T}$ spectra for energies $\sqrt{s_{N N}}=11.5,19.6,39 \mathrm{GeV}$ at $60 \%-80 \%$ centrality range.

\section{Elliptic flow}

The studies of the elliptic flow were preformed in order to examine the description of matter expansion included in EPOS model. The data simulated with the EPOS were compared with STAR results published in Ref. 12. The studies of beam energy were performed at mid-rapidity region for $p, \bar{p}, \pi^{+}, \pi^{-}, K^{+}$, $K^{-}$at $\sqrt{s_{N N}}=11.5,19.6,39 \mathrm{GeV}$ in minimum bias $(0-80 \%)$ using $\eta$-method. Particles with $|\eta|<1$, momentum between 0.15 and $5 \mathrm{GeV} / \mathrm{c}$ were taken into consideration.

The calculated values for pions and kaons coming from 


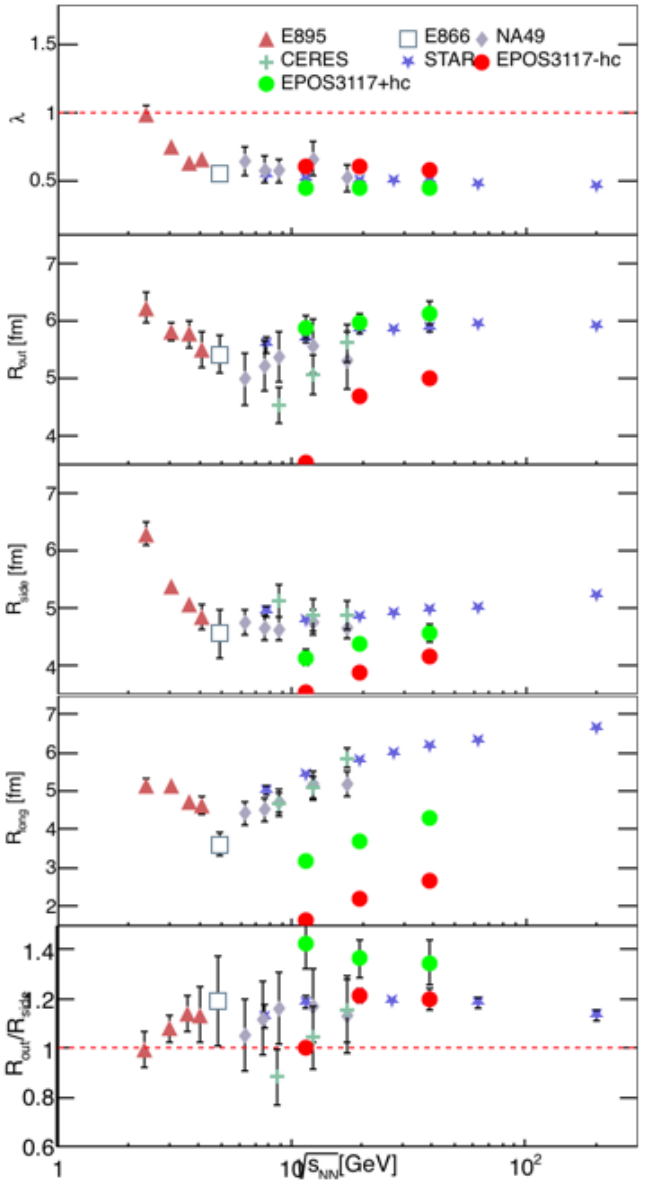

Fig. 8. $\lambda$ and radii dependence on energy $\sqrt{s_{N N}}$ for different experiments and EPOS data $\left(k_{T} \approx 0.225 \mathrm{GeV} / \mathrm{c}\right)$. [17]

simulation are slightly higher than expected. Although for $p_{T}>1.2 \mathrm{GeV}$ and $\sqrt{s_{N N}}=11.5 \mathrm{GeV}$ the pions flow is low. The pions are about $90 \%$ of all produced particles in the event, so they have a strong impact on the shape of $p_{T}$ spectra shown before. The low flow could result in the discrepancies in the slopes of the spectras. The same tendency is visible for antiprotons. The elliptic flow of protons in all $p_{T}$ bins are comparable for both data sets.

\section{CONCLUSION}

The EPOS model predictions for $\mathrm{Au}+\mathrm{Au}$ collisions at BES energy spectrum are verified with results obtained with experimental data. The $p_{T}$ spectra, correlation functions for identical pions and elliptic flow at $11.5,19.6$ and $39 \mathrm{GeV}$ for both simulated and STAR data were compared and presented. Observed discrepancies can be caused by too small impact of particles origin in core. The transparency of the collision should be investigated as well. The work is still in progress.

\section{ACKNOWLEDGMENT}

I would like to express my huge gratitude for my supervisor Dr Hanna Zbroszczyk, who from the beginning has been supportive and inspiring. My work would not be possible without Professor Klaus Werner. I really appreciate the whole

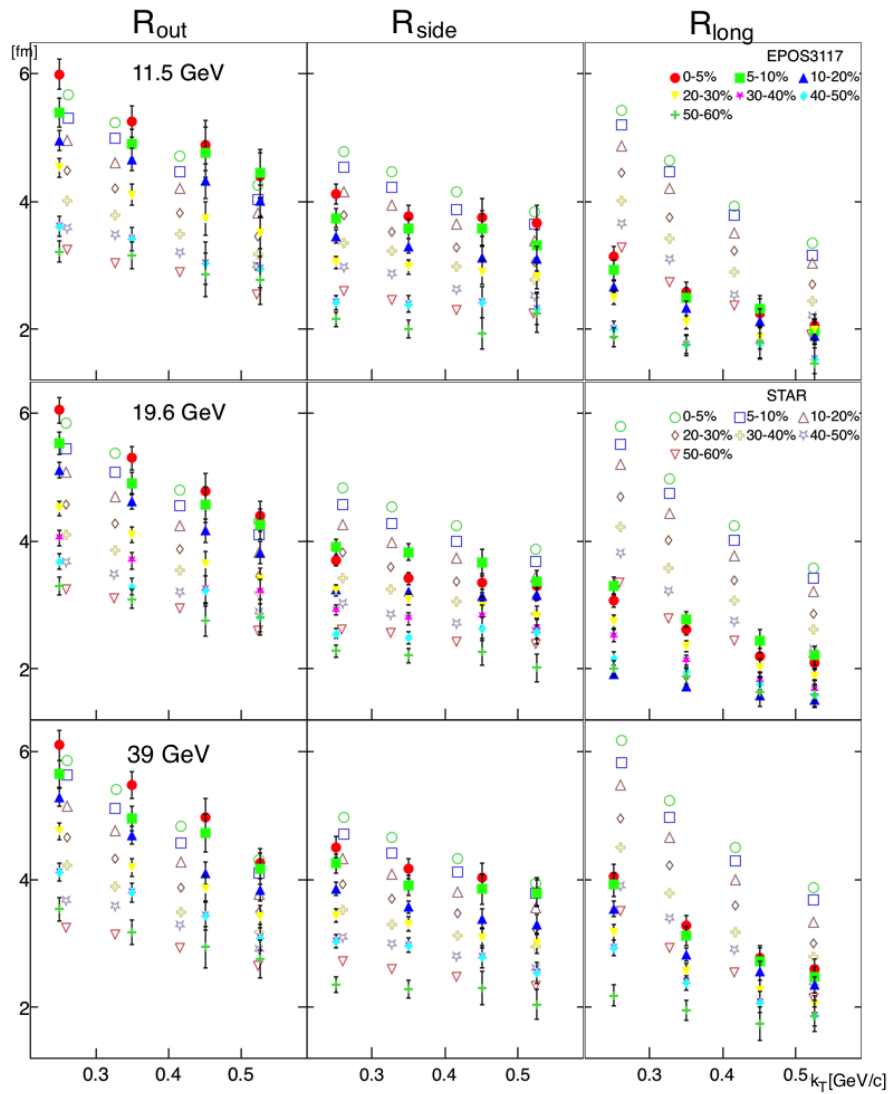

Fig. 9. Radii calculated for various centralities and $k_{T}$ bins [17]

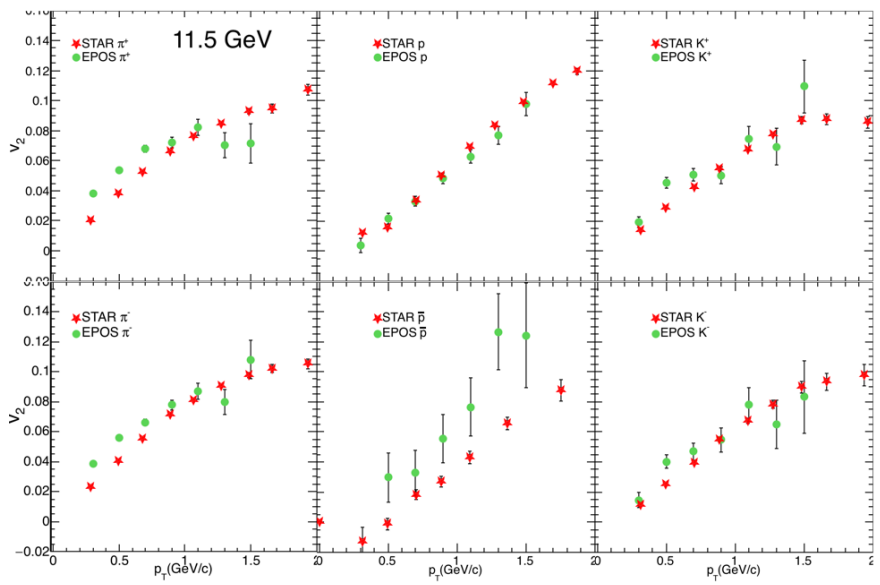

Fig. 10. Elliptic flow dependence on $p_{T}, \sqrt{s_{N N}}=11.5 \mathrm{GeV}$ [12].

time we spent on the discussions, which have been crucial in this research. Many valuable advice helped me to develop my physics knowledge. I am also really thankful to his $\mathrm{PhD}$ student Gabriel Sophys for all offered help and advice. I would like to extend my gratitude for support to the whole Heavy-Ion Reaction Group at Faculty of Physics at Warsaw University of Technology and to Subatech laboratory.

This work was supported by the Grants: UMO2012/07/D/ST2/02123 supported by National Science Centre, Poland. 


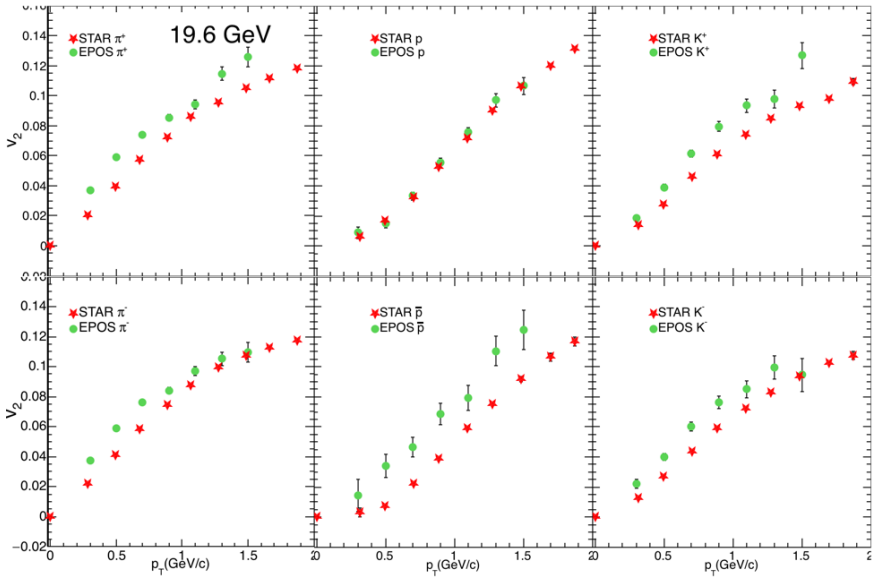

Fig. 11. Elliptic flow dependence on $p_{T}, \sqrt{s_{N N}}=19.6 \mathrm{GeV}$ [12].

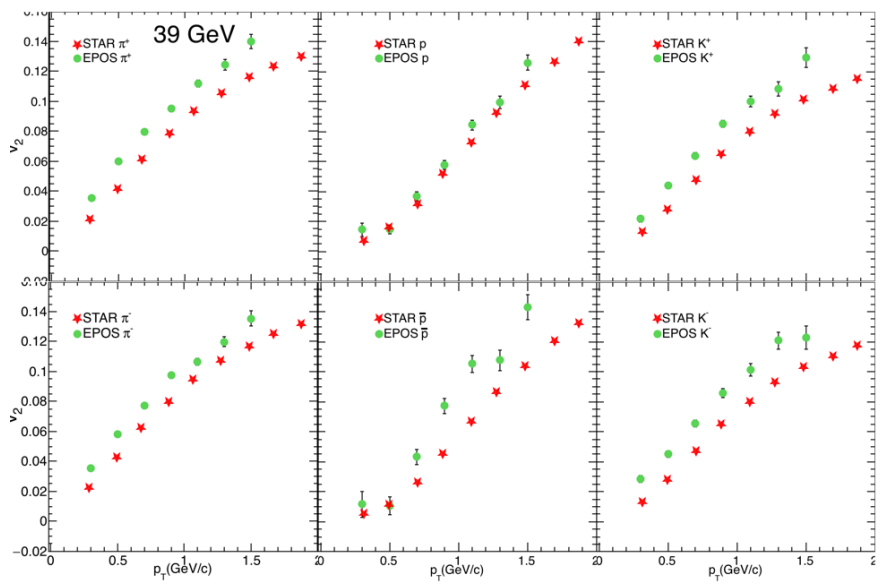

Fig. 12. Elliptic flow dependence on $p_{T}, \sqrt{s_{N N}}=39 \mathrm{GeV}$ [12].

\section{REFERENCES}

[1] D. H. J., H. M., O. S., P. T., and W. K., "Parton based Gribov-Regge theory," Phys. Rept., vol. 350, pp. 93-289, 2001.

[2] G. Odyniec, "Results and future of beam energy scan program at RHIC," XI Workshop on Particle Correlations and Femtoscopy, Warsaw 2015.

[3] D. Cebra, "Exploring the qcd phase diagram: RHIC beam energy scan II," Presentation on Low Energy RHIC electron Cooling Review, Brookhaven National Laboratory, Upton, NY, 2013.

[4] U. Gordon Baym, University of Illinois, "Hanbury brown twiss intensity interferometry: from stars to nuclei to atoms and electrons."

[5] M. A. Lisa, S. Pratt, R. Soltz, and U. Wiedemann, "Femtoscopy in relativistic heavy ion collisions," Ann. Rev. Nucl. Part. Sci., vol. 55, pp. 357-402, 2005.

[6] G. F. Bertsch, "Pion Interferometry as a Probe of the Plasma," Nucl. Phys., vol. A498, pp. 173C-180C, 1989.

[7] H. Tilsner, "Two-particle correlations at 40-A-GeV, 80-A-GeV, and 158A-GeV Pb - Au collisions," Ph.D. dissertation, Heidelberg U., 2002.

[8] D. H. Rischke and M. Gyulassy, "The Time-Dealy Signature of QuarkGluon-Plasma Formation in Relativistic Nuclear Collisions," 1996.

[9] H. Masui, A. Schmah, and A. M. Poskanzer, "Event plane resolution correction for azimuthal anisotropy in wide centrality bins," Nucl. Instrum. Meth., vol. A833, pp. 181-185, 2016.

[10] J. Lidrych, "Femtoscopy with unlike-sign kaons at the STAR experiment," 5th International Conference on New Frontiers in Physics Kolymbari, Crete, Greece.

[11] V. S. A., P. A. M., and S. Raimond, "Collective phenomena in noncentral nuclear collisions," 2008.

[12] A. L. et al., "Elliptic flow of identified hadrons in $\mathrm{Au}+\mathrm{Au}$ collisions at $\sqrt{s_{N N}}=7.7-62.4 \mathrm{GeV}$ " Phys. Rev., vol. C88, p. 014902, 2013.

[13] W. Klaus, L. Fu-Ming, and P. Tanguy, "Parton ladder splitting and the rapidity dependence of transverse momentum spectra in deuteron-gold collisions at RHIC," Phys. Rev., vol. C74, p. 044902, 2006.

[14] M. Anderson, B. Pennington, "The Lund string model." 1985.

[15] W. K., K. Iu., B. M., P. T., and P.-H. S., "Jets, Bulk Matter, and their Interaction in Heavy Ion Collisions at Several TeV," Phys. Rev., vol. C85, p. 064907, 2012.

[16] H. S. P., "Measurement of beam energy dependent nuclear modification factors at STAR," J. Phys. Conf. Ser., vol. 446, p. 012017, 2013.

[17] A. L. et al., "Beam-energy-dependent two-pion interferometry and the freeze-out eccentricity of pions measured in heavy ion collisions at the STAR detector," Phys. Rev., vol. C92, no. 1, p. 014904, 2015. 